\title{
Standardization and quantification is a key to the future of atherosclerosis FDG PET/CT imaging
}

\author{
Darko Pucar, MD, PhD, ${ }^{\mathrm{a}}$ and Chi Liu, $\mathrm{PhD}^{\mathrm{b}}$ \\ a Department of Radiology \& Biomedical Imaging; Section of Nuclear Medicine, Yale University \\ School of Medicine, New Haven, CT \\ b Department of Radiology \& Biomedical Imaging, Biomedical Engineering, Yale University, \\ New Haven, CT
}

Received Sep 17, 2019; accepted Sep 17, 2019

doi: $10.1007 / \mathrm{s} 12350-019-01905-7$

\section{See related article, pp. 1349-1359}

The current clinical nuclear medicine positron emission tomography imaging (PET) practice is dominated by oncologic imaging for cancer staging, restaging, and treatment monitoring. Some practices also conduct PET neuroimaging (dementia, seizures) and cardiac imaging (perfusion, viability, and inflammation studies). Vascular PET imaging is an exciting research tool, with currently limited clinical applications beyond detection of vasculitis.

The overarching idea of PET vascular imaging is that molecular imaging will be supplemental to anatomic imaging in risk stratification, outcome prediction, and treatment guidance of vascular disorders. The interest is particularly high for vascular PET imaging of atherosclerosis due to its impact on population morbidity and mortality. ${ }^{1}$

Considerable progress in the prevention of atherosclerosis-related acute coronary syndrome (ACS) and stroke has been made by risk factors modifications and medical and invasive therapies advances. Nevertheless, current preventive measures are only partially effective and further improvement in the prevention of atherosclerosis and its consequences is clearly needed. Atherosclerosis is characterized by accumulation of

Reprint requests: Darko Pucar, MD, PhD, Department of Radiology \& Biomedical Imaging; Section of Nuclear Medicine, Yale University School of Medicine, 333 Cedar Street, New Haven, CT 06511, USA; darkolpucar@outlook.com

J Nucl Cardiol 2021;28:1360-3.

$1071-3581 / \$ 34.00$

Copyright (c) 2019 American Society of Nuclear Cardiology. lipids, inflammatory cells, and connective tissue in the arterial walls with subsequent formation of atherosclerotic plaques. ${ }^{2,3}$ The rupture of vulnerable plaque initiates clot formation with subsequent thrombosis and infarction. At present, the risk of plaque rupture cannot be reliably predicted in the individual patients. However, since the arterial inflammation has a critical role in plaque rapture, imaging of vascular inflammation may identify patients at high risk for vascular events. ${ }^{4}$

${ }^{18} \mathrm{~F}$-fludeoxyglucose (FDG) is a glucose analogue that is taken into the cells by glucose transporters and phosphorylated by hexokinase. The resulting molecule ${ }^{18}$ F-FDG-6-phosphate misses 2-hydroxyl group needed for glycolysis and it is metabolically trapped in the cell allowing PET imaging. Increased glycolysis and FDG accumulation is observed in inflammation and malignancy, including plaque inflammation. ${ }^{5,6}$ Unfortunately, at least in United States, with the exception of vasculitis imaging, FDG PET utilization for vascular inflammation is limited to research only. Center for Medicare \& Medicaid Services (CMS) does not cover FDG PET imaging for infection and inflammation. This brings the key question on the future of vascular inflammation imaging and FDG PET/CT in particular. Can vascular FDG PET/CT ever become a mainstream imaging defined as being routinely used for initial risk stratification, re-stratification, and management of atherosclerotic diseases of coronary, carotid, and other vascular territories and reimbursed for these indications? Alternatively, will FDG PET/CT always remain a research tool for understanding of atherosclerosis pathogenesis and drug development? The answer to this burning question may well lay with the vascular molecular imaging quantification and standardization.

The history of oncologic FDG PET/CT imaging may provide a glimpse in the future of vascular FDG 
PET/CT imaging. High tumor-to-background ratio and superior tumor assessment to anatomic imaging in oncologic FDG PET/CT was an undisputed fact for many tumors for decades. Yet, existing data were not enough to convince CMS to cover oncologic PET/CT studies. Thus, coverage with evidence development (CED) under National Oncologic PET Registry (NOPR) was approved by CMS in 2006. NOPR is an internetbased USA-wide prospective registry that was made available for enrollment to all PET facilities that are participating providers in Medicare program. The primary research goal was to assess the effect of PET on referring physicians' plans of intended patient management across the spectrum of expanded cancer indications (diagnosis, staging, restaging, suspected recurrence, and treatment monitoring). ${ }^{7}$ The NOPR was managed by American College of Radiology (ACR) through ACR Imaging Network (ACRIN) and allowed participating PET facilities to use their institutional routine acquisition and interpretation protocols. NOPR project was successful, with PET changing intended management in $36.5 \%$ of cases, ${ }^{8}$ and with CMS deciding to cover FDG PET imaging for a majority of oncologic indications. Subsequent analysis of NOPR data discovered great variability in PET reporting, with only $72 \%$ of studies reporting Standardized Uptake Values (SUVs). ${ }^{9}$ Although rigorous standardization and quantification may have not been necessary to demonstrate the overall value of oncologic PET, their significance cannot be overstated. In fact, FDG PET/CT still remains in the shadows of CT for tumor treatment response evaluation since the universally accepted PET equivalent of CTBased Response Evaluation Criteria in Solid Tumors (RECIST) ${ }^{10}$ is still lacking. Even when the assessment is visual as in lymphoma treatment response, it was necessary to standardize FDG PET interpretation relative to blood pool and liver references (Deauville scale), before PET could take a central stage in the most recent Lugano Classification for lymphoma assessment. ${ }^{11}$

Unfortunately, high target to background ratio (TBR) is not a luxury of vascular inflammation FDG PET. Vascular activity is usually subtle and generally ignored by oncologic PET readers. Thus, vascular imaging had to be quantitative from the inception, and this is exemplified by the recent position paper of the Cardiovascular Committee of the European Association of Nuclear Medicine (EANM) on PET imaging of atherosclerosis. ${ }^{12}$ This important work was motivated by a desire to overcome the limitations imposed by current variety of FDG PET protocols used for arterial inflammation imaging. The hope was that standardized PET protocol will allow comparison between studies and creation of large multicenter registries. The proposed protocol standardized the requirements for FDG dose, injection-to-imaging time, prescan glucose value, imaging time and reconstruction, vascular territories to be analyzed, and FDG uptake parameters. The most important requirement is delaying PET acquisition from 1 hour used in oncologic imaging to 2 hour after FDG injection to allow sufficient FDG accumulation in the arterial wall and to reduce the intensity of FDG signal in the blood. ${ }^{6,13,14}$ Since atherosclerotic plaques are small, their PET avidity is subject to partial volume effect (PVE) with background signal from the circulating blood. However, delayed imaging of $>3$ hour from injection does not provide any additional improvement in the quantification of FDG uptake in the vessel wall. ${ }^{6,13,14}$ It should be noted that added value of delayed imaging (typically 2-3 hour) versus early imaging is a matter of substantial and unresolved controversy in oncologic imaging, with a potential superiority of delayed imaging in some settings such as prognostication of lung cancer or detection of recurrent brain tumor after treatment as recently reviewed by Houshmand et al. ${ }^{15}$ Thus, current 1-hour imaging delay is the most logistically convenient but not necessarily the best even for oncologic PET imaging. Another important requirement for atherosclerosis PET imaging is a blood glucose level $\leq 7 \mathrm{nmol} / \mathrm{L}$, although the impact of glucose level on FDG uptake in atherosclerosis is not well studied. One study observed increased blood pool activity with hyperglycemia, with a negative correlation between prescan glucose levels and SUV and TBR in the ascending aorta and carotid arteries. ${ }^{14}$ The recommended acquisition time of 8 minutes per bed position for vascular imaging is typically 2-3 times longer than that for oncologic imaging. In terms of quantification, EANM position paper advised on using TBR instead of SUV and comparing arterial FDG uptake only in the same vascular territory. Mean $\mathrm{TBR}_{\text {mean }}$ and $\mathrm{TBR}_{\text {max }}$ of the entire vascular territory was suggested to be used in prospective or retrospective studies assessing the correlation of arterial FDG uptake and clinical risk factors or studies identifying the patients at high risk for cardiovascular events in oncologic population. In retrospective studies more limited analysis of common carotid, ascending aorta, and aortic arch territories was proposed, while in prospective studies additional proposed territories were abdominal aorta, iliac, and femoral arteries. Most Diseased Segment (MDS) Analysis $\left(\mathrm{TBR}_{\max }\right.$ in a small segment of the index vessel with the highest FDG uptake) and active segment analysis (only arterial locations with a high predefined FDG uptake at baseline analyzed) were reserved for high-risk common carotid lesions and interventional studies using arterial inflammation as a surrogate endpoint. ${ }^{16,17}$

In this issue of JNC, Lawal et al aimed to evaluate the impact of applying EANM position paper 
recommendations on PET quantification of arterial ${ }^{18} \mathrm{~F}$ FDG uptake. Fifty-seven patients were prospectively imaged at 60 minutes (range 60-69 minutes) using routine oncologic ${ }^{18}$ F-FDG PET protocol (early imaging) and at $\geq 120$ minutes (range 120-245 minutes) using the EANM position paper protocol (delayed imaging). The patients were adults who underwent FDG $\mathrm{PET} / \mathrm{CT}$ for various oncologic indications and fever of unknown origin, had no pathology on their scans including calcified atherosclerotic plaques, had glucose $\leq 7 \mathrm{mmol} / \mathrm{L}$, and did not have active malignancy or renal failure, nor used anti-inflammatory drugs. The vascular territory evaluated was the ascending aorta and the parameters analyzed were aortic SUV max, superior vena cave (SVC) SUV mean, and target to background ratio. TBR was defined as aortic SUV max/SVC SUV mean and was obtained for early and delayed imaging. A single reader analyzed the images. Aortic SUV max increased by a mean of $70 \%$ while SVC SUV mean decreased by a mean of $52 \%$ between early and delayed imaging $(P<.001)$. TBR increased by $122 \%$ following delayed imaging. TBR increased while SVC SUV mean declined across all delayed time-points. Aortic SUV max significantly increased at delayed time-points between 120 and 180 minutes. No significant increase in aortic SUV max was seen at delayed time-points beyond 180 minutes. The authors concluded that applying EANM position paper protocol leads to an improved quantification of vascular inflammation through an increase in the quantified vascular tracer uptake and decrease in blood pool background activity.

The study supports use of the EANM position paper protocol which is its principal message. The utility of any guideline critically depends on the willingness of clinical and scientific community to provide testing and if possible validation in the practice. Despite limitations discussed below, the dramatic improvement of the ascending aorta TBR on delayed optimized vascular imaging relative to standard oncologic imaging is striking. In test-retest setting with each patient serving as his/her own control, there is little doubt on the superiority of optimized vascular protocol over routine oncologic protocol in this vascular territory. Although similar observations have been previously reported, the study does provide further insight on the utility of extending time to delayed imaging beyond 2 hour, potentially to 3 hour. It should be kept in mind, that any prolongation of injection-to-imaging time may worsen the already difficult logistic prospects of delayed imaging for either vascular or oncologic indications.

Since the study was conducted in the setting of oncologic imaging rather as a dedicated vascular study, rigorous cardiovascular risk stratification could not be performed. By virtue of the exclusion criteria, it is reasonable to assume that a low-risk population was analyzed. However, this also limits the applicability of the study to such population. Since only delayed imaging was optimized for vascular imaging, the effect of time delay may have been amplified by other shortcomings of early imaging. The control of delayed imaging time was poor due to logistic reasons in a busy clinic, which resulted in wide spread of delayed imaging times limiting the power of the study. For example, 14, 29,7 , and 7 patients were imaged at 120, 121-150, 151180 , and $>180$ minutes, respectively, resulting in very small samples for each time group. A more robust design in which delayed imaging would be obtained at 2 hour only or at 2 and 3 hour was not possible. Since only ascending aorta was analyzed, the results cannot be automatically extrapolated to other vascular territories. Since the population has no atherosclerotic plaques, only TBR parameter could be analyzed, and thus the results cannot be extrapolated to the most diseased or active segment analysis. Considering previously reported low interobserver variability in quantitative vascular PET imaging, only one imaging interpreter was utilized in the study. This is in contrast to ACR study quality recommendations which advocate for use of at least 2 independent readers. This is important since vascular PET imaging was not tested outside of research settings by more general and less experienced imaging readers. Thus, low interobserver variability in vascular PET imaging may not hold in the general radiology practice, particularly considering very subtle nature of arterial vascular abnormalities and time pressure of real life. Thus, it is important for the future vascular PET studies to include multiple readers with variable experience, so that the required level of training can be defined.

In the study by Lawal et al, the image reconstruction parameters for the delayed imaging are generally consistent with those in the EANM position paper, including OSEM with 120 iterations and without postfiltering. The EANM position paper also recommended using a 1-mm voxel size for image reconstruction, which might not be feasible depending on the scanner vendor. For example, the Philips Vereos scanner allows 1-mm voxel size reconstruction, ${ }^{18}$ while the smallest reconstruction voxel size for the Siemens mCT scanner is $2 \mathrm{~mm}$. For the early imaging in the study by Lawal et al, the reconstruction parameters were quite different, with only 8 iterations for OSEM and a 5-mm FWHM Gaussian filter. While it is understandable that such parameters are typical for oncology studies, the discrepancy might have affected the interpretation of the results in this paper. For iterative reconstruction, it is well established that larger number of iterations will improve resolution, contrast, but also increase image noise that could artifactually increase the $\mathrm{SUV}_{\max }$ 
measurement. ${ }^{19,20}$ Therefore, it is hard to make a fair comparison in TBR and SUV between the early and late imaging scans without using the same reconstruction parameters. In particular, the difference between 8 iterations and 120 iterations is considerably large, and the 5$\mathrm{mm}$ Gaussian filtering applied on the early scan but not on the delayed scan will only amplify this difference. Therefore, the TBR and SUV measurements between the early and late imaging scans in this study could be confounded by the different reconstruction and filtering parameters. It is vital to match such parameters to isolate the investigation solely on the potential value of delayed imaging.

To conclude, although FDG PET atherosclerosis imaging has unequivocal potential as a biomarker for cardiovascular risk and treatment intervention effects, many challenges lay ahead. It is difficult to conduct a high-quality research-level studies in the setting of limited funding and scanner availability. Nevertheless, only such studies will allow generation of robust data on clinical value of vascular FDG PET that will establish appropriateness. For vascular PET to transform from a promising research tool to clinical reality, only the best is good enough.

\section{Disclosure}

Darko Pucar and Chi Liu have nothing to disclose.

\section{References}

1. Benjamin EJ, Muntner P, Alonso A, Bittencourt MS, Callaway CW, Carson AP, et al. Heart disease and stroke statistics-2019 update: A Report From the American Heart Association. Circulation 2019;139:e56-528.

2. Miller YI, Chang MK, Binder CJ, Shaw PX, Witztum JL. Oxidized low density lipoprotein and innate immune receptors. Curr Opin Lipidol 2003;14:437-45.

3. Skalen K, Gustafsson M, Rydberg EK, Hulten LM, Wiklund O, Innerarity TL, et al. Subendothelial retention of atherogenic lipoproteins in early atherosclerosis. Nature 2002;417:750-4.

4. Rudd JH, Hyafil F, Fayad ZA. Inflammation imaging in atherosclerosis. Arterioscler Thromb Vasc Biol 2009;29:1009-16.

5. Figueroa AL, Subramanian SS, Cury RC, Truong QA, Gardecki JA, Tearney GJ, et al. Distribution of inflammation within carotid atherosclerotic plaques with high-risk morphological features: A comparison between positron emission tomography activity, plaque morphology, and histopathology. Circ Cardiovasc Imaging 2012;5:69-77.

6. Tawakol A, Migrino RQ, Bashian GG, Bedri S, Vermylen D, Cury $\mathrm{RC}$, et al. In vivo $18 \mathrm{~F}$-fluorodeoxyglucose positron emission tomography imaging provides a noninvasive measure of carotid plaque inflammation in patients. J Am Coll Cardiol 2006;48:181824.
7. Hillner BE, Liu D, Coleman RE, Shields AF, Gareen IF, Hanna L, et al. The National Oncologic PET Registry (NOPR): Design and analysis plan. J Nucl Med 2007;48:1901-8.

8. Hillner BE, Siegel BA, Liu D, Shields AF, Gareen IF, Hanna L, et al. Impact of positron emission tomography/computed tomography and positron emission tomography (PET) alone on expected management of patients with cancer: Initial results from the National Oncologic PET Registry. J Clin Oncol 2008;26:2155-61.

9. Coleman RE, Hillner BE, Shields AF, Duan F, Merlino DA, Hanna LG, et al. PET and PET/CT reports: Observations from the National Oncologic PET Registry. J Nucl Med 2010;51:158-63.

10. Eisenhauer EA, Therasse P, Bogaerts J, Schwartz LH, Sargent D, Ford R, et al. New response evaluation criteria in solid tumours: Revised RECIST guideline (version 1.1). Eur J Cancer 2009;45:228-47.

11. Cheson BD, Fisher RI, Barrington SF, Cavalli F, Schwartz LH, Zucca E, et al. Recommendations for initial evaluation, staging, and response assessment of Hodgkin and non-Hodgkin lymphoma: The Lugano classification. J Clin Oncol 2014;32:3059-68.

12. Bucerius J, Hyafil F, Verberne HJ, Slart RH, Lindner O, Sciagra R, et al. Position paper of the Cardiovascular Committee of the European Association of Nuclear Medicine (EANM) on PET imaging of atherosclerosis. Eur $\mathrm{J}$ Nucl Med Mol Imaging 2016;43:780-92.

13. Blomberg BA, Thomassen A, Takx RA, Hildebrandt MG, Simonsen JA, Buch-Olsen KM, et al. Delayed (1)(8)F-fluorodeoxyglucose PET/CT imaging improves quantitation of atherosclerotic plaque inflammation: Results from the CAMONA study. J Nucl Cardiol 2014;21:588-97.

14. Bucerius J, Mani V, Moncrieff C, Machac J, Fuster V, Farkouh ME, et al. Optimizing 18F-FDG PET/CT imaging of vessel wall inflammation: The impact of $18 \mathrm{~F}-\mathrm{FDG}$ circulation time, injected dose, uptake parameters, and fasting blood glucose levels. Eur $\mathbf{J}$ Nucl Med Mol Imaging 2014;41:369-83.

15. Houshmand S, Salavati A, Segtnan EA, Grupe P, Hoilund-Carlsen PF, Alavi A. Dual-time-point imaging and delayed-time-point Fluorodeoxyglucose-PET/computed tomography imaging in various clinical settings. PET Clin 2016;11:65-84.

16. Emami H, Vucic E, Subramanian S, Abdelbaky A, Fayad ZA, Du S, et al. The effect of BMS-582949, a P38 mitogen-activated protein kinase (P38 MAPK) inhibitor on arterial inflammation: A multicenter FDG-PET trial. Atherosclerosis 2015;240:490-6.

17. Tawakol A, Fayad ZA, Mogg R, Alon A, Klimas MT, Dansky H, et al. Intensification of statin therapy results in a rapid reduction in atherosclerotic inflammation: Results of a multicenter fluorodeoxyglucose-positron emission tomography/computed tomography feasibility study. J Am Coll Cardiol 2013;62:909-17.

18. Zhang J, Maniawski P, Knopp MV. Performance evaluation of the next generation solid-state digital photon counting PET/CT system. EJNMMI Res 2018;8:97.

19. Tong S, Alessio AM, Kinahan PE. Noise and signal properties in PSF-based fully 3D PET image reconstruction: An experimental evaluation. Phys Med Biol 2010;55:1453-73.

20. Liu C, Alessio A, Pierce L, Thielemans K, Wollenweber S, Ganin $\mathrm{A}$, et al. Quiescent period respiratory gating for PET/CT. Med Phys 2010;37:5037-43.

Publisher's Note Springer Nature remains neutral with regard to jurisdictional claims in published maps and institutional affiliations. 